\title{
On the spider fauna (Arachnida: Aranei) of the 'Ust'-Belsk' Natural Park and its vicinities
}

\section{Материалы к фауне пауков (Arachnida: Aranei) природного парка "Усть-Бельск" и его окрестностей}

\author{
A.N. Sozontov ${ }^{1,2}$, S.L. Esyunin ${ }^{3}$ \\ A.Н. Созонтов ${ }^{1,2}$, С. $\Lambda$. Есюнин $^{3}$ \\ ${ }^{1}$ Department of Ecology of Animals, Udmurt State University, Universitetskaya Street 1/1, Izhevsk 426034 Russia.
Кафедра экологии животных, Удмуртский государственный университет, ул. Университетская, 1/1, Ижевск 426034 Россия.
${ }^{2}$ State Zoo of Udmurtia, Kirova Street 8, Izhevsk 426033 Russia.
БУК УР “Зоопарк Удмуртии”, ул. Кирова, 8, Ижевск 426033 Россия.
${ }^{3}$ Department of Zoology, Perm State University, Bukireva Street 15, Perm 614600 Russia.
Кафедра зоологии беспозвоночных животных, Пермский государственный университет, ул. Букирева 15, Пермь 614600 Россия. \\ KEY WORDS: spider, fauna, Udmurtia, 'Ust'-Belsk' Natural Park. \\ КЛЮЧЕВЫЕ СЛОВА: пауки, фауна, Удмуртия, природный парк “Усть-Бельск”.
}

ABSTRACT. A list of 160 spider species of 20 families collected from the 'Ust'-Belsk' Natural Park and its neighbouring area (Karakulino District, Udmurt Republic) is provided. Two spider families, Hahniidae and Mimetidae, and 45 species are reported from Udmurtia for the first time. Faunistic records and habitat preferences for all species and data on distribution of the newly recorded ones are provided.

РЕЗЮМЕ. Приводится список 160 видов пауков из 20 семейств, собранных в природном парке “Усть-Бельск" и прилегающей к нему территории (Каракулинский район, Удмуртская Республика). Два семейства, Hahniidae и Mimetidae, и 45 видов впервые указаны для фауны пауков Удмуртии. Даны точки находок и данные по местообитаниям всех видов и распространение новых для региона видов.

\section{Introduction}

The araneofauna of Udmurt Republic has been intensively studied during recent years [Sozontov, Esyunin, 2012; Sozontov, 2012a,b, 2013] and currently includes 209 species. In the present paper we have provided new faunistic data from several localities of Karakulino District of Udmurtia situated on the territory of the 'Ust'-Belsk' Natural Park or its vicinities (see Map). The vicinities are those localities that currently do not belong to the Natural Park but have their own status of 'monuments of nature' and thus have already been recommended for inclusion in the expanded version of the park [Baranova et al., 2011]; numbers $1-3$ in the list of localities given below under 'Material and Methods'.
To date, no special arachnological studies have been done in Karakulino District. The record of Eresus kollari Rossi, 1846 from Bima River [Zubko, 2001] needs confirmation [Sozontov, Dedyukhin, 2012]. In 20112012, we collected about 2000 spider specimens belonging to160 species, of which 45 species are new to the fauna of Udmurtia; in the following list these species are marked with asterisks * and are provided with data on their global distribution. Yet, two families Hahnidae and Mimetidae - have been reported for Udmurt Republic for the first time. Thus, as of now, the total diversity of Udmurtian araneofauna is 254 species of 26 families.

The aim of this paper is to present a faunistic list of spiders of the 'Ust'-Belsk' Natural Park with details of their habitat preferences.

\section{Material and methods}

The 'Ust'-Belsk' Natural Park is located in Karakulino District, on the south border of Udmurt Republic. Its territory lies in the fluvial plain of the lower reaches of Kama River and belongs to the broadleaved-coniferous forest subzone of the subboreal zone, with a noticeable influence of the forest-steppe. Yet, a rather large part of the district has been turned to agricultural fields. Although such human impact has slackened during last decades, the forest areas have significantly decreased already which resulted in a more noticeable steppification.

This paper is based on the spider collections made by the first author (not marked in the following text) and by S.V. Dedukhin (marked in the text as SVD) from the several localities of the 'Ust'-Belsk' Natural 


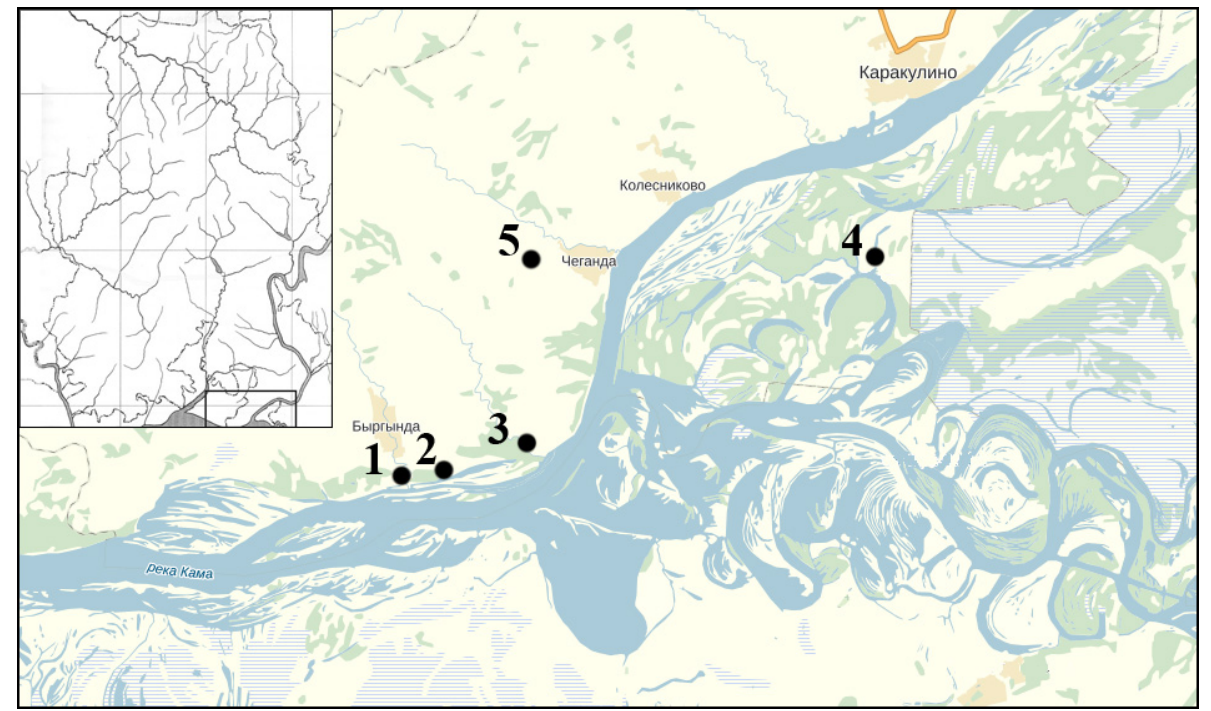

Map. Collecting localities (1-5; see for the explanations given in 'Materials and methods').

Карта. Локалитеты (1-5; описание см. в разделе 'Материал и методы').

Park and its vicinities in 2011-2012. Detailed information of the collecting localities is given below (see Map):

1 - Byrgynda Vil. (55 $\left.53^{\prime} \mathrm{N}, 53^{\circ} 25^{\prime} \mathrm{E}\right)$, 'Byrgyndinsk' stow; recommended for inclusion in the natural park.

2 - Shygnanda Vil. $\left(55^{\circ} 53^{\prime} \mathrm{N}, 53^{\circ} 27^{\prime} \mathrm{E}\right)$, vicinities of 'Ust'-Belsk' stow; recommended for inclusion in the natural park.

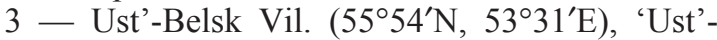
Belsk' stow; recommended for inclusion in the natural park.

4 - Malyashy Lake $\left(55^{\circ} 57^{\prime} \mathrm{N}, 53^{\circ} 41^{\prime} \mathrm{E}\right)$, 'Ust'Belsk' Natural Park (in its current limits).

5 - Chegnanda Vil. $\left(55^{\circ} 57^{\prime} \mathrm{N}, 53^{\circ} 31^{\prime} \mathrm{E}\right)$, vicinities of 'Ust'-Belsk' Natural Park; already included in the natural park.

The typology of species ranges follows Esyunin \& Marusik [2011].

The studied material is shared between the collections of the Udmurt State University, Izhevsk, Russia (UdSU) and the Department of Zoology of the Perm State University, Perm, Russia (PSU).

Abbreviations used in the text: SVD - S.V. Dedyukhin, juv. — juvenile specimens.

\section{Annotated list of species}

\section{ARANEIDAE}

Araneus angulatus Clerck, 1757

MATERIAL. $1 \sigma^{7}, 2$ ㅇ, [3], inundated broad-leaved forest, flood-plain of Kama River, 6-10.VII.2011.

Araneus diadematus Clerck, 1757

MATERIAL. 1 juv., [3], river flood-plain, 4-10.VII.2011; 1 juv., [3], Populus tremula-Tilia forest with oak and Acer, 3.VIII.2012.
Araneus marmoreus Clerck, 1757

MATERIAL. 1 subadult +, [3], flood-plain of Kama River, 610.VII.2011.

Araneus quadratus Clerck, 1757

MATERIAL. $1 \bigcirc^{\top}, 1$ subadult,+ 1 juv., [3], dry meadow on slope, flood-plain of Kama River, 6-10.VII.2011.

Araniella proxima (Kulczyński, 1885)

MATERIAL. $1 \sigma^{7}$, [5], multiherbaceous dry meadows on slope, 16.VI.2012.

Argiope bruennichi (Scopoli, 1772)

MATERIAL. $1 \sigma^{7}$, [1], dry lichen-grass association on wood side of inundated mixed forest, 4.VIII.2012.

Cercidia prominens (Westring, 1851)

MATERIAL. $2 O^{7} \sigma^{7}, 1$ 우 [1], dry lichen-grass association on sand, inundated Quercus-forest, 12-21.VI.2012; 1 subadult + , [3], flood-plain of Kama River, 6-10.VII.2011; 1 juv., [3], Populus tremula-Tilia forest with oak and Acer, 3.VIII.2012.

Cyclosa conica (Pallas, 1772)

MATERIAL. $1 \sigma^{7}$, [2], river flood-plain, 11-20.VI.2012.

Hypsosinga pygmaea (Sundevall, 1831)

MATERIAL. 1 +, [3], dry meadow, 7.VII.2011; 1 \$, [4], inundated meadow, 11.VI.2011.

Larinioides folium (Schrank, 1803)

MATERIAL. $80^{7} \sigma^{7}, 11$ 우, [2], multiherbaceous meadows, banks of former river-bed, 11-20.VI.2012; $1 \bigcirc^{\top}, 4$ 우, [3], dry meadow on slope, banks of former river-bed, flood-plain of Kama River, 6-10.VII.2011; 1 9. [3], inundated broad-leaved forest, 13.VI.2012; 1 + [3], limestone denudation, 3.VIII.2012; $3 \bigcirc^{\top} \sigma^{\top}, 5$ 우 [4], banks of former river-bed, inundated meadow, 11.VI.2011.

Larinioides ixobolus (Thorell, 1873)

MATERIAL. $1 \sigma^{7}, 1$ ㅇ, [2], flood-plain of Kama River, 1120.VI.2012; 1 O$^{7}$, [3], river flood-plain, 6-10.VII.2011. 
Larinioides patagiatus (Clerck, 1757)

MATERIAL. $2 \sigma^{7} \sigma^{7}, 19$, [2], banks of former river-bed, 12 20.VI.2012; $1 \mathrm{O}^{7}, 3$ OP, [3], banks of former river-bed, flood-plain of Kama River, 6-10.VII.2011; $1 \sigma^{\top}$, [3], limestone denudation, 3.VIII.2012; $1 \mathrm{O}^{\top}, 3$ 우, [4], banks of former river-bed, 11.VI.2011.

Mangora acalypha (Walckenaer, 1802)

MATERIAL. 1 +, [3], ravine, 9.VII.2011, leg. SVD; 2 +क, [5], multiherbaceous dry meadows, 16.VI.2012.

*Neoscona adianta (Walckenaer, 1802)

MATERIAL. $3 \sigma^{7} \sigma^{7}, 1$ \%, [2], multiherbaceous meadows, 15.VI.2012; 1 O$^{7}$, [5], edge of route, 16.VI.2012.

DISTRIBUTION. Trans-Palaearctic nemoral range.

Singa hamata (Clerck, 1757)

MATERIAL. 2 우, 2juv., [3], flood-plain of Kama River, 410.VII.2011; 2 우, [4], banks of former river-bed, 11.VI.2011.

Singa nitidulaC.L. Koch, 1844

MATERIAL. 4 우, 2 subadult 우, [3], banks of former riverbed and Kama River, 6-10.VII.11.

\section{CHEIRACANTHIIDAE}

Cheiracanthium erraticum (Walckenaer, 1802)

MATERIAL. 5 우, [2], bank of former river-bed, 1120.VI.2012; $1 \sigma^{7}, 4$ Oᄋ+ [3], dry meadow, flood-plain of Kama River, 4-10.VII.2011; $1 \mathrm{O}^{2}$, [4], bank of former river-bed, 11.VI.11; 12 우, [5], edge of route, 16.VI.2012.

Cheiracanthium pennyi O. Pickard-Cambridge, 1873 MATERIAL. $1 \mathrm{O}^{7}$, [3], ravine, 9.VII.2011, leg. SVD.

\section{CLUBIONIDAE}

Clubiona frisia Wunderlich et Schuett, 1995

MATERIAL. 1 + , [3], flood-plain of Kama River, 610.VII.2011

*Clubiona germanica Thorell, 1871

MATERIAL. 1 ․ [3], no habitat data, 6-10.VII.2011.

DISTRIBUTION. West-Central Palaearctic temperate range.

Clubiona lutescens Westring, 1851

MATERIAL. 1 9, [3], bank of former river-bed, 6-10.VII.2011.

Clubiona neglecta O. Pickard-Cambridge, 1862

MATERIAL. 2 +\$, [2], multiherbaceous meadows, 15.VI.2012.

*Clubiona pallidula (Clerck, 1757)

MATERIAL. 2 or $^{7}$, [1], inundated Quercus-forest, 1220.VI.2012; 1 \%, [2], no habitat data, 11-20.VI.2012; 1 , [3], ravine, 9.VII.2011, leg. SVD; 2 O$^{7} \sigma^{7}$, [3], Populus tremula-Tilia forest with oak and Acer, 11-20.VI.12.

DISTRIBUTION. Trans-Palaearctic temperate range.

*Clubiona phragmitis C.L. Koch, 1843

MATERIAL. 7 우, [2], bank of former river-bed, 12 20.VI.2012; $1 \mathrm{O}^{7}, 1$ \% , [3], bank of former river-bed, 6-10.VII.2011; 1 + , [4], bank of former river-bed, 11.VI.2011.

DISTRIBUTION. Trans-Palaearctic nemoral range.
Clubiona stagnatilis Kulczyñski, 1897

MATERIAL. 2 우, [2], bank of former river-bed, 1220.VI.2012

Clubiona reclusa O. Pickard-Cambridge, 1863

MATERIAL. 1 \%, [3], flood-plain of Kama River, 410.VII.2011.

\section{CORINIIDAE}

Phrurolithus festivus (C.L. Koch, 1835)

MATERIAL. $1 \sigma^{7}, 1$,,$[1]$, inundated Quercus-forest, pitfalltraps, 12-20.VI.2012; 1 + 1 subadult + , [2], Populus tremulaTilia forest with oak and Acer, 14.VI.2012; 1 , , [3], Populus tremula-Tilia with oak and Acer, 11-20.VI.2012; 7 +P, [3], dry and sunny bank of former river-bed, 2-5.VIII.2012.

\section{DICTYNIDAE}

Argenna subnigra (O. Pickard-Cambridge, 1861)

MATERIAL. 1 +, [4], inundated meadow, 11.VI.2011.

Dictyna arundinacea (Linnaeus, 1758)

MATERIAL. 6 +O, [2], multiherbaceous meadows, bank of former river-bed, 11-20.VI.2012; 3 कQ, [3], bank of former riverbed, flood-plain of Kama River, 4-10.VII.2011.

*Dictyna major Menge, 1869

MATERIAL. 2 우, [3], flood-plain of Kama River, 610.VII.2011.

DISTRIBUTION. Circum-Holarctic temperate range.

Dictyna pusilla Thorell, 1856

MATERIAL. 1 क, [3], flood-plain of Kama River, 610.VII.2011; 3 우, [4], bank of former river-bed, 11.VI.2011.

Dictyna uncinata Thorell, 1856

MATERIAL. 4 우, [3], flood-plain of Kama River, 610.VII.2011.

*Emblyna annulipes (Blackwall, 1846)

MATERIAL. 1 \%, [2], no habitat data, 11-20.VI.2012.

DISTRIBUTION. Circum-Holarctic temperate range.

\section{GNAPHOSIDAE}

*Drassodes pubescens (Thorell, 1856)

MATERIAL. $1 \sigma^{7},[1]$, dry lichen-grass association on sand, 12-21.VI.2012; 1 ○ึ, [2], inundated meadow, 12-20.VI.2012; 1 $\sigma^{7}$, [3], inundated broad-leaved forest, 12-20.VI.2012.

DISTRIBUTION. Trans-Palaearctic nemoral range.

Drassodes villosus (Thorell, 1856)

MATERIAL. 2 우, [3], flood-plain of Kama River, 610.VII.2011.

*Drassyllus lutetianus (L. Koch, 1866)

MATERIAL. 5 O $^{\top} \sigma^{7}, 3$ 우, [1], dry lichen-grass association on sand, inundated Quercus-forest, 12-21.VI.2012; 6 ○ $^{7}, 3$ 우, [2], inundated meadow, Populus tremula-Tilia forest with oak and Acer, 11-20.VI.2012; 1 9, [3], inundated meadow, 6-10.VII.2011; $1 \mathrm{O}^{\text {T }, 4}$ 우, [3], inundated broad-leaved forest, 12-20.VI.2012; 2 우, [3], 
bank of former river-bed, 2-5.VIII.2012; 2 Oొ $^{\top}, 2$ 우, [4], inundated meadow, 11.VI.2011.

DISTRIBUTION. West-Palaearctic nemoral range.

*Drassyllus praeficus (L. Koch, 1866)

MATERIAL. $1 \bigcirc^{7}$, [2], inundated meadow, 12-20.VI.2012.

DISTRIBUTION. West-Central-Palaearctic temperate range.

Drassyllus pusillus (C.L. Koch, 1833)

MATERIAL. 1 , [1], dry lichen-grass association on sand, 12-21.VI.2012; $10^{7}$, 2 क्+, [3], inundated meadow, 6-10.VII.2011;

* Gnaphosa lugubris (C.L. Koch, 1839)

MATERIAL. $3 \sigma^{7} \sigma^{7}, 1$ 9, [3], flood-plain of Kama River, 610.VII.2011.

DISTRIBUTION. West-Central-Palaearctic nemoral range.

*Haplodrassus cognatus (Westring, 1861)

MATERIAL. 2 우, [2], Populus tremula-Tilia forest with oak and Acer, 11-20.VI.2012.

DISTRIBUTION. Euro-West Siberia temperate range.

Haplodrassus pseudosignifer Marusik, Hippa et Koponen, 1996

MATERIAL. $3 \bigcirc^{7} O^{7}, 1+$, [1], dry lichen-grass association on sand, inundated Quercus-forest, 12-21.VI.2012; $1 \sigma^{7}$, [2], floodplain of Kama River, 11-21.VI.2012; $3 \sigma^{\top} \sigma^{7}$, [3], inundated and dry meadows, 6-10.VII.2011.

Haplodrassus silvestris (Blackwall, 1833)

MATERIAL. $3 \sigma^{7} \sigma^{7}, 1 \%$, [1], inundated Quercus-forest, $12-$ 20.VI.2012; 1 \%, [1], Populus tremula-Tilia forest with oak and Acer, 4.VIII.2012; $3 \bigcirc^{7} \bigcirc^{7}, 2$ 우, [2], Populus tremula-Tilia forest with oak and Acer, 11-20.VI.2012; 1 +, [4], inundated meadow, 11.VI.2011.

Haplodrassus soerenseni (Strand, 1900)

MATERIAL. 1 ㅇ, [1], inundated Quercus-forest, 12 20.VI.2012.

* Micaria formicaria (Sundevall, 1831)

MATERIAL. $2 \sigma^{7} \sigma^{7}$, [2], flood-plain of Kama River, 1121.VI.2012; 2 O $^{7} \sigma^{7}$, [3], flood-plain of Kama River, 4-10.VII.2011; 2 우, [5], dry meadow, 16.VI.2012.

DISTRIBUTION. Trans-Palaearctic nemoral range.

Micaria pulicaria (Sundevall, 1831)

MATERIAL. 3 우, [4], inundated meadow, bank of former river-bed, 11.VI.2011.

*Zelotes latreillei (Simon, 1878)

MATERIAL. $1 \sigma^{7}$, [2], inundated meadow, 12-20.VI.2012; 1 , [3], inundated meadow, 6-10.VII.2011; 2 우, [3], bank of former river-bed, 2-5.VIII.2012; 1 +, [4], inundated meadow, 11.VI.2011.

DISTRIBUTION. West-Palaearctic temperate range.

*Zelotes longipes (L. Koch, 1866)

MATERIAL. 1 , [1], dry lichen-grass association on sand, 12-21.VI.2012; $1 \sigma^{7},[3]$, open and sunny bank of former riverbed, 2-5.VIII.2012.

DISTRIBUTION. West-Central Palaearctic nemoral range.
Zelotes subterraneus (C.L. Koch, 1833)

MATERIAL. $1 \sigma^{7}$, [1], dry lichen-grass association on sand, 12-21.VI.2012; 3 우, [2], Populus tremula-Tilia forest with oak and Acer, 11-20.VI.2012; $1 \bigcirc^{7}$, [3], inundated broad-leaved forest, 12-20.VI.2012; 1 क , [3], bank of former river-bed, 25.VIII.2012.

\section{*HAHNIIDAE}

*Hahnia ononidum Simon, 1875

MATERIAL. 2 우, [2], Populus tremula-Tilia forest with oak and Acer, Pinus forest with low-density grassy, 14.VI.2012.

DISTRIBUTION. Trans-Palaearctic-West Nearctic temperate range.

\section{LINYPHIIDAE}

Abacoproeces saltuum (L. Koch, 1872)

MATERIAL. $11 \sigma^{7} \sigma^{\top}, 2$ 우, [1], dry lichen-grass association on sand, inundated Quercus-forest, 12-21.VI.2012; 10 O $^{\top} \mathrm{O}^{\mathrm{T}}$, 3 우, [2], Populus tremula-Tilia forest with oak and Acer, Pinus forest with low-density grassy, 11-20.VI.2012; $60^{7} \sigma^{7}$, [3], inundated broad-leaved forest, 12-20.VI.2012; 2 우, [3], Populus tremula-Tilia forest with oak and Acer, bank of former river-bed, 2-5.VIII.2012.

*Agyneta mollis (O. Pickard-Cambridge, 1871)

MATERIAL.2 $\sigma^{7} \sigma^{7}$, [2], no habitat data, 11-20.VI.2012.

DISTRIBUTION. Trans-Palaearctic-West Nearctic temperate range.

*Agyneta saaristoi Tanasevitch, 2000

MATERIAL. $20^{7} O^{7}, 2$ 우, [2], no habitat data, 11-20.VI.2012.

DISTRIBUTION. West-Central Palaearctic nemoral range.

Bolyphantes alticeps (Sundevall, 1833)

MATERIAL.1 subadult + , [3], flood-plain of Kama River, 610.VII.2011.

* Cnephalocotes obscurus (Blackwall, 1834)

MATERIAL.1 O', [4], inundated meadow, 11.VI.2011.

DISTRIBUTION. Circum-Holarctic temperate range.

*Diplocephalus picinus (Blackwall, 1841)

MATERIAL. $10 \bigcirc^{7} \sigma^{7},[1]$, dry lichen-grass association on sand, inundated Quercus-forest, 12-21.VI.2012; 1 +, [2], no habitat data, 11-21.VI.2012.

DISTRIBUTION. West-Palaearctic temperate range.

Diplostyla concolor (Wider, 1834)

MATERIAL.1 $\sigma^{7}$, [1], inundated Quercus-forest, 12-20.VI.2012; $1 \mathrm{O}^{\top}, 2$ + 9 , [2], Populus tremula-Tilia forest with oak and Acer, 1120.VI.2012; $1 \mathrm{O}^{7}$, [3], inundated broad-leaved forest, 12-20.VI.2012; $3 \mathrm{O}^{\top} \mathrm{O}^{\top}, 1$,, [3], bank of former river-bed, 2-5.VIII.2012.

* Gnathonarium dentatum (Wider, 1834)

MATERIAL. $1 \sigma^{7}$, [2], flood-plain of Kama River, 1120.VI.2012.

DISTRIBUTION. Trans-Palaearctic temperate range. 
*Gongylidiellum murcidum Simon, 1884

MATERIAL. $2 \bigcirc^{7} \sigma^{7}, 2$, 2 , [2], no habitat data, 11-20.VI.2012.

DISTRIBUTION. West-Central Palaearctic nemoral range

Helophora insignis (Blackwall, 1841)

MATERIAL. 1 subadult + , [2], Populus tremula-Tilia fores with oak and Acer, 14.VI.2012; 5 subadult $O^{7} \sigma^{7}, 5$ subadult of [3], flood-plain of Kama River, 6-10.VII.11; 2 subadult $\sigma^{7} \sigma^{7}, 1$ subadult + , [3], Populus tremula-Tilia forest with oak and Acer, bank of former river-bed, 2-5.VIII.2012.

Hypomma bituberculatum (Wider, 1834)

MATERIAL. 2 우, [2], inundated meadow, 11-20.VI.2012.

* Hypomma fulvum (Bösenberg, 1902)

MATERIAL. 2 우, [2], bank of former river-bed, 12-20.VI 2012.

DISTRIBUTION. Trans-European temperate range.

Linyphia tenuipalpis Simon, 1884

MATERIAL. $10^{7}, 1$ \% , [1], dry lichen-grass association on sand, 4.VIII.2012; 4 subadult $\bigcirc^{7} \sigma^{7}$, [3], slope, dry meadow, 610.VII.11.

Linyphia triangularis (Clerck, 1757)

MATERIAL. 1 subadult $\sigma^{7}$, [2], Populus tremula-Tilia forest with oak and Acer, 14.VI.2012; $1 \mathrm{O}^{2}, 3$ subadult $\mathrm{O}^{7} \mathrm{O}^{2}$, 1 subadult + , [3], bank of former river-bed, inundated and flood-

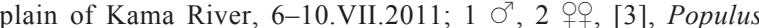
tremula-Tilia forest with oak and Acer, bank of former riverbed, 2-5.VIII.2012.

* Maso sundevalli (Westring, 1851)

MATERIAL. $2 \sigma^{\top} \sigma^{7}, 1$; , [1], dry lichen-grass association on sand, 12-21.VI.2012; 1 , , [2], no habitat data, 11-21.VI.2012.

DISTRIBUTION. Circum-Holarctic temperate range. 1871)

*Microlinyphia impigra (O. Pickard-Cambridge,

MATERIAL. 1 \%, [3], flood-plain of Kama River, on vegetation, 3.VIII.2012.

DISTRIBUTION. Circum-Holarctic temperate range.

Microneta viaria (Blackwall, 1841)

MATERIAL. $10^{7}, 8$ 우, [2], Populus tremula-Tilia forest with oak and Acer, 11-21.VI.2012; 1 +, [3], bank of former river-bed, 2-5.VIII.2012

Neriene emphana (Walckenaer, 1841)

MATERIAL. $10^{7}, 11$ +o, [3], flood-plain of Kama River, 610.VII.2011.

Neriene montana (Clerck, 1757)

MATERIAL. 1 ㅇ, [2], inundated, 11-20.VI.2012; 2 우, [3], inundated broad-leaved forest, 12-20.VI.2012; 1 , [3], inundated, 6-10.VII.2011.

*Palliduphantes alutacius (Simon, 1884)

MATERIAL. 1 +, [3], Pinus forest without herbaceous, 35.VIII.2012.

DISTRIBUTION. Euro-West Siberian nemoral range.
Panamomops mengei Simon, 1926

MATERIAL. $2 \sigma^{7} \sigma^{7}$, [1], dry lichen-grass association on sand, 12-21.VI.2012; 2 O $^{7} O^{7}, 4$ OO , [2], Populus tremula-Tilia forest with oak and Acer, Pinus forest with low-density grassy, 11-21.VI.2012.

*Pelecopsis mengei (Simon, 1884)

MATERIAL. $10^{\top 7}, 2$ 우, [2], Populus tremula-Tilia forest with oak and Acer, inundated meadow, 12-20.VI.2012.

DISTRIBUTION. Circum-Holarctic temperate range.

Porrhomma pygmaeum (Blackwall, 1834)

MATERIAL. 1 O', [2], inundated, 11-20.VI.2012.

*Tapinopa longidens (Wider, 1834)

MATERIAL. 1 O', 1 juv., [3], Pinus forest without herbaceous, 3-5.VIII.2012.

DISTRIBUTION. West-Palaearctic nemoral range.

Tenuiphantes nigriventris (L. Koch, 1879)

MATERIAL. $1 \sigma^{2},[3]$, Populus tremula-Tilia forest with oak and Acer, 3.VIII.2012.

Tenuiphantes tenebricola (Wider, 1834)

MATERIAL. $1 \sigma^{2}$, [3], Pinus forest without herbaceous, 35.VIII.2012.

*Thyreostenius parasiticus (Westring, 1851)

MATERIAL. 2 우, [3], open and sunny bank of former riverbed, 2-5.VIII.2012.

DISTRIBUTION. Circum-Holarctic temperate range. 1878)

*Walckenaeria atrotibialis (O. Pickard-Cambridge,

MATERIAL. $1 \sigma^{\top}, 1$, , [2], no habitat data, 11-21.VI.2012.

DISTRIBUTION. Circum-Holarctic temperate range.

*Walckenaeria nudipalpis (Westring, 1851)

MATERIAL. 1 9, [2], Populus tremula-Tilia forest with oak and Acer, 14.VI.2012.

DISTRIBUTION. Trans-Palaearctic temperate range.

*Walckenaeria vigilax (Blackwall, 1853)

MATERIAL. $10^{7}$, [2], Populus tremula-Tilia forest with oak and Acer, 11-20.VI.2012.

DISTRIBUTION. West Palaearctic-West Nearctic disjunctive temperate range.

\section{LIOCRANIDAE}

Agroeca cuprea Menge, 1873

MATERIAL. 1 + , [3], ravine, 9.VII.2011, leg. SVD.

*Agroeca lusatica (L. Koch, 1875)

MATERIAL. 1 , [1], dry lichen-grass association on sand, 12-21.VI.2012.

DISTRIBUTION. West-Palaearctic nemoral range.

Agroeca proxima (O. Pickard-Cambridge, 1871)

MATERIAL. $4 O^{7} \sigma^{7}$, [3], Pinus forest without herbaceous, 35.VIII.2012. 


\section{LYCOSIDAE}

Alopecosa cuneata (Clerck, 1757)

MATERIAL. 1 ․ [1], dry lichen-grass association on sand, 11-20.VI.2012.

Arctosa cinerea (Fabricius, 1777)

MATERIAL. 1 q, [3], flood-plain of Kama River, 610.VII.2011.

*Arctosa leopardus (Sundevall, 1833)

MATERIAL. $2 O^{7} O^{7}, 1$ ㅇ, [3], bank of former river-bed, 610.VII.2011; $2 \mathrm{O}^{7} \mathrm{O}^{7}$, [4], bank of former river-bed, 11.VI.2011.

DISTRIBUTION. West-Central Palaearctic nemoral range.

*Arctosa stigmosa (Thorell, 1875)

MATERIAL. 3 우, [3], flood-plain of Kama River, 610.VII.2011.

DISTRIBUTION. West-Palaearctic nemoral range.

Pardosa fulvipes (Collett, 1876)

MATERIAL. $3 \sigma^{7} \sigma^{7}, 4$ OP, [3], 7.VII.11, dry and inundated meadows, 4-10.VII.2011; 1 O , 3 우, [4], inundated meadow, 11.VI.2011.

Pardosa lugubris (Walckenaer, 1802)

MATERIAL. $4 \bigcirc^{7} O^{7}, 4 \circ 9$, [3], inundated broad-leaved forest, inundated and flood-plain of Kama River, 4-10.VII.2011; $5 \mathrm{O}^{7} \mathrm{O}^{7}$, 4 우, [4], Populus tremula-Tilia forest with oak and Acer, inundated meadow, 11.VI.2011.

Pardosa paludicola (Clerck, 1757)

MATERIAL. 4 $+9,[3]$, inundated meadow, flood-plain of Kama River, 6-10.VII.2011.

Pardosa palustris (Linnaeus, 1758)

MATERIAL. $4 \mathrm{O}^{7} \sigma^{7}, 2$ 우, [3], inundated meadow, 610.VII.2011

Pardosa plumipes (Thorell, 1875)

MATERIAL. $11 O^{7} \sigma^{7}$, [3], inundated meadow, 6-10.VII.2011; $1 \bigcirc^{\top}$, [4], bank of former river-bed, 11.VI.2011.

Pardosa prativaga (L. Koch, 1870)

MATERIAL. 3 ơ , [3], bank of former river-bed, 610.VII.2011; $5 O^{7} O^{7}, 4$ Q, , [4], bank of former river-bed, inundated meadow, 11.VI.2011.

Pirata piraticus (Clerck, 1757)

MATERIAL. $3 \sigma^{7} \sigma^{7}, 19,[3]$, inundated broad-leaved forest, flood-plain of Kama River, 6-10.VII.2011; 1 ○7, 1 , [4], bank of former river-bed, 11.VI.2011.

Pirata piscatorius (Clerck, 1757)

MATERIAL. 1 q, [3], inundated broad-leaved forest, 9.VII.2011, leg. SVD; 1 +, [4], inundated meadow, 11.VI.2011.

Piratula hygrophilus (Thorell, 1872)

MATERIAL. 1 \%, [2], inundated, 11-21.VI.2012; 2 O $^{7} \sigma^{7}, 4$ of , [3], bank of former river-bed, flood-plain of Kama River, 610.VII.2011.

Trochosa ruricola (De Geer, 1778)

MATERIAL. 3 య $^{7}, 1$, [4], inundated meadow, 11.VI.2011.
Xerolycosa miniata (C.L. Koch, 1834)

MATERIAL. 2 OొT $^{7}$, [3], inundated meadow, 6-10.VII.2011; 1 ㅇ, [3], bank of former river-bed, 2-5.VIII.2012; 2 우, [4], inundated meadow, 11.VI.2011; $4 \bigcirc^{\top} \bigcirc^{7}, 4$ 우, [3], slope, dry meadow, 6-10.VII.2011.

\section{*MIMETIDAE}

*Ero furcata (Villers, 1789)

MATERIAL. 1 +, [3], Pinus forest without herbaceous, 35.VIII.2012

DISTRIBUTION. Trans-Palaearctic temperate range.

\section{OXYOPIDAE}

Oxyopes sp.

MATERIAL. 1 juv., [5], slope, dry meadow, 16.VI.2012.

\section{PHILODROMIDAE}

Philodromus cespitum (Walckenaer, 1802)

MATERIAL. $1+$, [1], dry lichen-grass association on sand, 4.VIII.2012; $13 \mathrm{O}^{7} \mathrm{O}^{\top}, 21$ Oᄋ , [2], multiherbaceous meadows, bank of former river-bed, 11-20.VI.2012; 10 कO+ [3], inundated and flood-plain of Kama River, 4-10.VII.2011; 1 +, [3], limestone denudation, 3.VIII.2012; $10^{7}, 8$ o+ , [4], inundated meadow, bank of former river-bed, 11.VI.2011; $3 \mathrm{O}^{2} \mathrm{O}^{2}, 6$ 우, [5], edge of route, dry meadow, 16.VI.2012.

Philodromus histrio (Latreille, 1819)

MATERIAL. 5 juv., [1], dry lichen-grass association on sand, 4.VIII.2012.

Philodromus poecilus (Thorell, 1872)

MATERIAL. 1 \%, [2], inundated, 11-20.VI.2012; 1 +, [4], bank of former river-bed, 11.VI.2011.

Thanatus formicinus (Clerck, 1757)

MATERIAL. 1 +, [3], dry meadow on slope, 7.VII.2011.

Thanatus sabulosus (Menge, 1875)

MATERIAL. $2 \sigma^{\top} \sigma^{\top}$, [1], dry lichen-grass association on sand, inundated Quercus-forest, 12-21.VI.2012; $1 \sigma^{7}$, [3], flood-plain of Kama River, south exposition, 6-10.VII.2011.

Tibellus maritimus (Menge, 1875)

MATERIAL. 1 +, [2], bank of former river-bed, 12-20.VI.2012.

Tibellus oblongus (Walckenaer, 1802)

MATERIAL. 3 +P, [1], dry lichen-grass association on sand, 4.VIII.2012; $3 \sigma^{7} \sigma^{7}, 6$ 우, [2], multiherbaceous meadows on slope, 11-20.VI.2012; $3 \mathrm{O}^{7} \mathrm{O}^{7}, 4$ +, , [3], dry meadow on slope, bank of former river-bed, inundated o Kama River, 4-10.VII.2011; 1 \%, [3], flood-plain of Kama River, on vegetation, 3.VIII.2012; $60^{7} \sigma^{7}$, 3 우, [4], inundated meadow, 11.VI.2011; $6 \bigcirc^{7} \bigcirc^{7}, 4$ 우, [5], dry meadow on slope, edge of route, 16.VI.2012.

\section{PISAURIDAE}

Dolomedes plantarius (Clerck, 1757)

MATERIAL. $1 \mathrm{O}^{\mathrm{T}},[2]$, bank of former river-bed, 12 20.VI.2012; 1 subadult,+ 5 juv., [3], flood-plain of Kama River, bank of former river-bed, 6-10.VII.2011; 1 juv., [4], inundated meadow, 11.VI.2011. 
Other MATERIAL. 1 , Udmurtia, Votkinsky District, Volkovsky Village, 10.VII.2012, leg. SVD; 1 \%, Udmurtia, Votkinsky District, Perevoznoe Village, "Siva" biological station, 7.VI.2013, leg. SVD.

REMARKS. This rare spider species was earlier recorded from Udmurtia by Zubko and Roshchinenko [1981], but without localities and habitat data.

Pisaura mirabilis (Clerck, 1757)

MATERIAL. 1 ㅇ, [3], inundated broad-leaved forest, 6.VII.2011.

\section{SALTICIDAE}

Ballus chalybeius (Walckenaer, 1802)

MATERIAL. 1 juv., [1], dry lichen-grass association on sand, 12-21.VI.2012; 1 subadult $\sigma^{7}$, [3], bank of former river-bed, 610.VII.2011.

Evarcha arcuata (Clerck, 1757)

MATERIAL. $5 O^{7} \sigma^{7}, 2$ 우, [1], dry lichen-grass association on sand, 4.VIII.2012; $3 \sigma^{7} \sigma^{\prime}$, [2], multiherbaceous meadows on slope, 11-20.VI.2012; $3 \bigcirc^{7} \sigma^{7}, 4$ OO , [3], dry meadow on slope, flood-plain of Kama River, 4-10.VII.2011; $1 \sigma^{7}$, 1 subadult $\sigma^{7}, 5$ 우, 1 subadul q, [4], inundated meadow, bank of former river-bed, 11.VI.2011; 1 $\sigma^{7}, 1$ subadult $\sigma^{7}$, [5], dry meadow on slope, 16.VI.2012.

Evarcha falcata (Clerck, 1757)

MATERIAL. $1 \sigma^{7}$, [1], Populus tremula-Tilia forest with oak and Acer, 4.VIII.2012; $1 \sigma^{7}, 5$ subadult $\sigma^{7} \sigma^{7}$, [3], dry meadow on slope, flood-plain of Kama River, 4-10.VII.2011; 2 juv., [4], bank of former river-bed, 11.VI.2011.

Heliophanus auratus C.L. Koch, 1835

MATERIAL. $1 \sigma^{7}, 4$ ㅇ, [2], bank of former river-bed, 1120.VI.2012; $3 O^{7} \sigma^{7}, 7$ OP , [3], dry meadow on slope, inundated and flood-plain of Kama River, bank of former river-bed, 4-11.VII.2011; $1 \sigma^{7}, 1$ \%, [3], limestone denudation, 3-4.VIII.2012; $2 \bigcirc^{7} \sigma^{7}$, [4], inundated meadow, 11.VI.2011.

Heliophanus flavipes (Hahn, 1832)

MATERIAL. 2 우, [3], dry meadow on slope, 7.VII.2011.

*Marpissa muscosa (Clerck, 1757)

MATERIAL. $10^{7}$, [1], dry meadow, 15.VIII.2011.

DISTRIBUTION. West-Palaearctic nemoral range.

* Marpissa radiata (Grube, 1859)

MATERIAL. 12 oq, [2], bank of former river-bed, 1220.VI.2012; 1 \%, [4], bank of former river-bed, 11.VI.2011.

DISTRIBUTION. West-Central Palaearctic nemoral range.

Phlegra fasciata (Hahn, 1826)

MATERIAL. $2 \sigma^{7} \sigma^{7}$, [1], dry lichen-grass association on sand, 12-21.VI.2012.

*Pseudicius encarpatus (Walckenaer, 1802)

MATERIAL. $4 \sigma^{7} \sigma^{7},[2]$, flood-plain of Kama River, 1120.VI.2012.

DISTRIBUTION. West-Palaearctic nemoral range.

REMARKS. This species, having West-Palaearctic nemoral range, in the Europe reaches to South Fennoscandia on the North. The given locality is the northeasternmost record of the species.
Sitticus dzieduszyckii (L. Koch, 1870)

MATERIAL. $5 \sigma^{7} \sigma^{7}, 4$ + , , [3], flood-plain of Kama River, 610.VII.2011; 1 ㅇ, [3], limestone denudation, 3.VIII.2012.

Sitticus floricola (C.L. Koch, 1837)

MATERIAL. 7 우, [2], bank of former river-bed, flood-plain of Kama River, 6-20.VI.12; 1 O', [3], flood-plain of Kama River, on vegetation, 3.VIII.2012; 1 \%, [4], bank of former river-bed, 11.VI.2011.

* Sitticus saltator (O. Pickard-Cambridge, 1868)

MATERIAL. $1 \sigma^{\curvearrowright}$, [1], dry lichen-grass association on sand, 12-21.VI.2012; 1 ㅇ, same locality and biotope, 4.VIII.2012; 2 $\mathrm{O}^{\top} \mathrm{O}^{7}$, [2], flood-plain of Kama River, 11-21.VI.2012.

DISTRIBUTION. West-Central Palaearctic nemoral range.

* Talavera aequipes (O. Pickard-Cambridge, 1871)

MATERIAL. 1 o, [2], flood-plain of Kama River, 1121.VI.2012.

DISTRIBUTION. West-Central-Palaearctic nemoral-steppe range.

\section{SPARASSIDAE}

Micrommata virescens (Clerck, 1757)

MATERIAL. $1 O^{7}, 1$, 2 juv., [2], multiherbaceous meadows on slope, bank of former river-bed, 11-20.VI.2012; 1 juv., [3], dry meadow on slope, 7.VII.2011; 2 juv., [3], Populus tremula-Tilia forest with oak and Acer, limestone denudation, 3.VIII.2012; 2 juv., [4], Populus tremula-Tilia forest with oak and Acer, inundated meadow, 11.VI.2011; 1 , 1 juv., [5], dry meadow on slope, edge of route, 16.VI.2012.

\section{TETRAGNATHIDAE}

Pachygnatha clercki Sundevall, 1823

MATERIAL. 1 + , [2], inundated, 11-20.VI.2012.

Tetragnatha dearmata Thorell, 1873

MATERIAL. $5 \sigma^{7} \sigma^{7}, 990$, [3], bank of former river-bed, inundated and flood-plain of Kama River, 4-10.VII.2011; $10^{7}, 1$, [4], bank of former river-bed, 11.VI.2011.

Tetragnatha extensa (Linnaeus, 1758)

MATERIAL. $4 O^{7} \sigma^{7}, 2$ 우, [3], dry meadow on slope, bank of former river-bed, flood-plain of Kama River, 6-10.VII.2011.

Tetragnatha montana Simon, 1874

MATERIAL. 2 ㅇ, [4], inundated meadow, bank of former river-bed, 11.VI.2011.

Tetragnatha pinicola $\mathrm{L}$. Koch, 1870

MATERIAL. 1 \%, [2], multiherbaceous meadows on slope, 15.VI.2012

Tetragnatha striata L. Koch, 1862

MATERIAL. 1 , , [2], bank of former river-bed, 12 20.VI.2012; 1 O$^{2}$, [3], flood-plain of Kama River, 8.VII.2011; 4 우, [3], flood-plain of Kama River, limestone denudation, on vegetation, 3.VIII.2012; 3 O $^{7}, 3$ +क, [4], flood-plain of Kama River, 11.VI.11.

Tetragnatha qiuae Zhu, Song et Zhang 2003

MATERIAL. $2 \sigma^{7} \sigma^{7}$, [2], bank of former river-bed, 12-

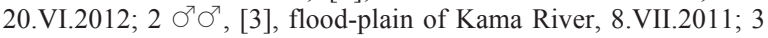


$0^{\top} \bigcirc^{\top}, 1$, [3], flood-plain of Kama River, limestone denudation, on vegetation, 3.VIII.2012.

REMARKS. T. qiuae was described from Central China (Tsinkhai Province) [Zhu et al., 2003]. This species occurs in Astrakhan' Region and South Kazakhstan [unpublished data]. Also the species occurs in Tuva and Mongolia [Yu.M. Marusik, pers. comm.]. It is likely that $T$. qiuae has a Central Asian range with an isolated population in the Middle Volga region. We assume that the species could have migrated from the north shore of Caspian Sea to Middle Volga region along the River Volga. A special paper devoted to this species is under preparation.

\section{THERIDIIDAE}

* Asagena phalerata (Panzer, 1801)

MATERIAL. 1 ○', [2], flood-plain of Kama River, 1121.VI.2012.

DISTRIBUTION. Trans-Palaearctic polyzonal range.

Crustulina guttata (Wider, 1834)

MATERIAL. $1 \sigma^{7}$, [1], dry lichen-grass association on sand, 12-21.VI.2012; $70^{7} \sigma^{7}, 22$ क्+, 7 subadult $\sigma^{7} \sigma^{7}, 29$ juv., [2], Рориlus tremula-Tilia forest with oak and Acer, 11-21.VI.2012; $10^{7}, 6$ 우, [3], bank of former river-bed, 2-5.VIII.2012.

*Crustulina sticta (O. Pickard-Cambridge, 1861)

MATERIAL. $1 \sigma^{7}$, [3], no habitat data, 6-10.VII.2011.

DISTRIBUTION. Circum-Holarctic temperate range.

Enoplognatha ovata (Clerck, 1757)

MATERIAL. 2 OP, [3], bank of former river-bed, flood-plain of Kama River, 6-10.VII.2011; 4 subadult $O^{\top} \sigma^{7}$, [4], inundated meadow, 11.VI.2011.

*Episinus angulatus (Blackwall, 1836)

MATERIAL. $10^{7}$, [4], inundated meadow, 11.VI.2011.

DISTRIBUTION. West-Palaearctic nemoral range.

Episinus truncatus Latreille, 1809

MATERIAL. 1 \%, [3], Pinus forest without herbaceous, 35.VIII.2012.

Euryopis flavomaculata (C.L. Koch, 1836)

MATERIAL. $4 \sigma^{7} \sigma^{7}, 1+$, [1], dry lichen-grass association on sand, inundated Quercus-forest, 12-21.VI.2012; $4 \bigcirc^{7} \sigma^{7}, 4$ 우, [2], Populus tremula-Tilia forest with oak and Acer, 11-20.VI.2012; 1 + , [3], inundated broad-leaved forest, 12-20.VI.2012; 1 \%, [3], Pinus forest without herbaceous, 3-5.VIII.2012.

Lasaeola tristis (Hahn, 1833)

MATERIAL. 1 + , [4], inundated meadow, 11.VI.2011.

Neottiura bimaculata (Linnaeus, 1767)

MATERIAL. $1 \sigma^{\top 7}$, [3], inundated, 4-10.VII.2011.

Parasteatoda lunata (Clerck, 1757)

MATERIAL. 1 q, [1], inundated Quercus-forest, 1220.VI.2012.

REMARKS. This rare spider species was earlier recorded from Udmurtia by Zubko and Roshchinenko [1981], but without localities and habitat data.
Parasteatoda ? tepidariorum (C.L. Koch, 1841)

MATERIAL. $1 \sigma^{\top}$, [2], no habitat data, 11-20.VI.2012; 3 우, [3], flood-plain of Kama River, 6-10.VII.2011.

REMARKS. The identification of this species causes some doubts, as our specimens may also belong to the closely related species Parasteatoda simulans (Thorell, 1875). Additional researches on the character variation in both species are required.

Parasteatoda tabulata (Levi, 1980)

MATERIAL. $2 \sigma^{7} \sigma^{7}, 1$, , [2], no habitat data, 11-20.VI.2012; $2 O^{7} \sigma^{7}, 1$ \%, [3], flood-plain of Kama River, 6-10.VII.2011.

Phylloneta impressa (L. Koch, 1881)

MATERIAL. $10 \odot^{7} \sigma^{7}, 6$ 우, [2], multiherbaceous meadows on slope, 11-20.VI.2012; 1 厂', 6 O+, [3], dry meadow on slope, ravine, flood-plain of Kama River, 7-9.VII.2011, SVD; $10^{7}, 2$ 우, [5], dry meadow on slope, edge of route, 16.VI.2012.

* Robertus arundineti (O. Pickard-Cambridge, 1871)

MATERIAL. 1 \%, [2], flood-plain of Kama River, 11-20.VI.12.

DISTRIBUTION. West-Central Palaearctic temperate range.

Robertus lividus (Blackwall, 1836)

MATERIAL. 1 o, [1], inundated Quercus-forest, 1220.VI.2012

Steatoda bipunctata (Linnaeus, 1758)

MATERIAL. 1 subadult + , 1 juv., [2], on wall, 11-20.VI.2012.

Steatoda castanea (Clerck, 1757)

MATERIAL. 1 subadult + , [2], inundated, 11-20.VI.2012.

Theridion pictum (Walckenaer, 1802)

MATERIAL. 1 +, [2], bank of former river-bed, 12-20.VI.2012; 1 , [3], flood-plain of Kama River, 6.VII.2011.

Theridion varians Hahn, 1833

MATERIAL. $10^{7}, 1$, [2], Pinus forest with low-density grassy, 11-20.VI.2012; 1 O', [3], flood-plain of Kama River, 610.VII.2011; $3 \bigcirc^{7} \sigma^{7}, 1$, [4], inundated meadow, bank of former river-bed, 11.VI.2011.

\section{THOMISIDAE}

Ebrechtella tricuspidata (Fabricius, 1775)

MATERIAL. $1 \mathrm{O}^{7}$, [1], inundated Quercus-forest, 1220.VI.2012; $3 \sigma^{7} \sigma^{7}, 1$ subadult $\sigma^{7}, 2$ juv., [1], dry lichen-grass association on sand, Populus tremula-Tilia forest with oak and

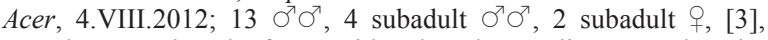
Populus tremula-Tilia forest with oak and Acer, limestone denudation, flood-plain of Kama River, bank of former river-bed, 25.VIII.2012; 1 ; , [4], inundated meadow, 11.VI.2011; 1 subadult +, [5], dry meadow on slope, 16.VI.12.

Misumena vatia (Clerck, 1757)

MATERIAL. 1 \%, [5], dry meadow on slope, 16.VI.12.

Ozyptila praticola (C.L. Koch, 1837)

MATERIAL. $36 \bigcirc^{7} \sigma^{7}, 21$ 우, [1], inundated Quercus-forest, dry lichen-grass association on sand, 12-21.VI.2012; $42 O^{7} O^{7}, 5$ O+P, [2], Populus tremula-Tilia forest with oak and Acer, 1121.VI.2012; $15 \mathrm{O}^{7} \sigma^{7}$, [3], Populus tremula-Tilia forest with oak and Acer, inundated broad-leaved forest, 12-20.VI.2012; $10^{7}$, [3], flood-plain of Kama River, south exposition, 6-10.VII.2011; $90^{7} \sigma^{\prime}$, [3], Pinus forest without herbaceous, Populus tremula-Tilia forest with oak and Acer, 3-5.VIII.2012. 
Table. Spider species diversity of Specially Protected Natural Reserves (SPNR) of the eastern part of Russian Plain (north to south).

Таблица. Видовое разнообразие пауков особо охраняемых природных территорий восточной части Русской Равнины (с севера на юг).

\begin{tabular}{|l|l|c|l|}
\hline \multicolumn{1}{|c|}{ SPNR } & \multicolumn{1}{|c|}{ Region } & $\begin{array}{c}\text { Species } \\
\text { number }\end{array}$ & \multicolumn{1}{|c|}{ Reference } \\
\hline 'Nurgush' Reserve & Kirov Region & 168 & Esyunin, Tselishcheva, 2011 \\
\hline 'Predural'e' Reserve & Perm Region & 334 & Esynin et al., 2011 \\
\hline 'Nechkinskiy' National park & Udmurt Republic & 160 & $\begin{array}{l}\text { Sozontov, Esyunin, 2012; Sozontov, unpublished } \\
\text { data }\end{array}$ \\
\hline 'Mariy Chodra' National park & Mari El Republic & 180 & Krasnobaev, 2004 \\
\hline 'Ust'-Belsk' Natural park & Udmurt Republic & 160 & Our data \\
\hline 'Volzhsko-Kamsk' Reserve & Tatarstan Republic & 240 & Krasnobaev, 2004 \\
\hline Mordovian Reserve & Mordovia Republic & 142 & Mikhailov, Trushina, 2013 \\
\hline 'Chavash Varmane' National park & Chuvash Republic & 161 & Krasnobaev, 2004 \\
\hline 'Zhiguli' Reserve & Samara Region & 113 & Krasnobaev, 2004 \\
\hline 'Samarskaya Luka' National Park & Samara Region & 335 & Krasnobaev, 2004 \\
\hline
\end{tabular}

*Ozyptila trux (Blackwall, 1846)

MATERIAL. 2 O $^{7}$, [1], inundated Quercus-forest, dry lichen-grass association on sand, 12-21.VI.2012; 3 O $^{\top} \mathrm{O}^{7}, 1$ +, [2], Populus tremula-Tilia forest with oak and Acer, inundated meadow, 11-20.VI.2012.

DISTRIBUTION. Trans-Palaearctic temperate range.

Tmarus piger (Walckenaer, 1802)

MATERIAL. $1 \sigma^{\top}$, [2], inundated, 11-20.VI.2012.

Xysticus bifasciatus C.L. Koch, 1837

MATERIAL. $1 \sigma^{7}, 1$ + , [3], inundated meadow, 6-10.VII.2011.

Xysticus cristatus (Clerck, 1757)

MATERIAL. 3 우, [3], dry meadow on slope, 6-10.VII.2011; 1 , [4], inundated meadow, 11.VI.2011; 2 of , [5], slope, dry meadow, 16.VI.2012.

Xysticus kochi Thorell, 1872

MATERIAL. 1 , [3], dry meadow on slope, 7.VII.2011.

Xysticus luctator L. Koch, 1870

MATERIAL. $23 \sigma^{\top} \sigma^{7}, 5$ 우, [1], dry lichen-grass association on sand, inundated Quercus-forest, 12-21.VI.2012; $25 \sigma^{7} \sigma^{7}, 2$ 우, [2], Populus tremula-Tilia forest with oak and Acer, 11-20.VI.2012; $13 \bigcirc^{\top} \sigma^{7}, 1$, [3], inundated broad-leaved forest, 12-20.VI.2012; 2 $\sigma^{7} \sigma^{7},[4]$, inundated meadow, 11.VI.2011;

Xysticus striatipes L. Koch, 1870

MATERIAL. $2 \sigma^{7} \sigma^{7}$, [1], dry lichen-grass association on sand, 4.VIII.2012; $1 \mathrm{O}^{\text {T, }}$ [2], multiherbaceous meadows, 4.VIII.2012.

\section{ZORIDAE}

Zora spinimana (Sundevall, 1833)

MATERIAL. $3 \sigma^{7} \sigma^{7}$, [2], inundated broad-leaved forest, inundated meadow, 12-20.VI.2012; 1 ○', [3], Populus tremula-Tilia forest with oak and Acer, 3.VIII.2012.

\section{Discussion}

The spider species diversity of the 'Ust'-Belsk' Natural Park is comparable with those of the majority of Specially Protected Natural Reserves (SPNR) lying eastward of the Russian Plain (see Table). However, it is $1.5-2$ times smaller than the spider diversity of the three best studied local faunas (Table).

The reasons for such disparity in the taxonomical diversity are not obvious. Indeed, local faunas differ in the state of their knowledge. However, a degree of such differences is difficult to estimate and therefore it is impossible to reveal an influence of this factor on the recorded diversity.

According to the available data (Table), there is no correlation between the species diversity and latitudinal zonation, as the richest faunas occur both in the north and in the south of the studied region. As shown by Esyunin [1995], the diversity of plain local araneofaunas of the Middle Urals varies within the limits of 270-290 species. Faunas with higher spider species diversity are usually characterized by a unique combination of landscape diversity and/or natural zones, as is the case of the 'Predural'e' Reserve [Esyunin et al., 2011].

It is worth noticing that the variation of diversity within the limits of 140-180 species seems to be most common. It can be argued that this score should reflect the number of species having high or average abundance in the studied communities that facilitate their capture. Long-term field studies and much larger collecting samples are required to record rarer species, which are likely to constitute at least a half of the overall species diversity of any local araneofauna. Usually, there is no need for recording rare species in order to draw a conclusion about the core of studied spider communities.

If the latter assumption is correct then a predictable spider diversity of the 'Ust'-Belsk' Natural Park should make up more than 300 species.

ACKNOWLEDGEMENTS. We are grateful to Dr S.V. Dedyukhin (Izhevsk, Russia) for his spider material and important remarks on this paper. We are also grateful Dr D.V. Logunov (Manchester, UK) for editing the English of the final draft. This project was funded by the Russian Foundation for Basic Research (grants No. 14-04-31178; 12-04-01548). 


\section{References}

Baranova O.G., Adakhovskiy D.A., Borisovskiy A.G., Dedyukhin S.V., Zubtsovskiy N.E., Perevoschikov A.A., Markova E.M., Rubtsova A.V., Tychinin V.A., Tyulkin Yu.A. 2011. [Rare and vanishing species of plants and animals from South part of Udmurtia and their conservation: results of scientific researches (2005-2009)]. Izhevsk: izd-vo "Udmurtskiy universitet". 272 pp. [in Russian]

Esyunin S.L. 1995. [Diversity of spider fauna in the Urals: basic trends and governing factors] // Uspechi sovremennoy biologii. Tom 115. Vyp.3. P.316-325 [in Russian, with English summary].

Esyunin S.L., Marusik Yu.M. 2011. [Experience of the classification of the areas of distribution for the Ural's spiders] // Vestnik Permskogo Universiteta. Biology. No.1. P.32-36 [in Russian, with English summary].

Esyunin S.L., Polyanin A.B., Vlasov P.I., Shulaeva E.A. 2011 [Spider fauna of "Predural'e" reserve (Perm Area): 40 years of researches] // Vestnik Permskogo universiteta. Vyp. 3-4. P. 16-23 [in Russian, with English summary].

Esyunin S.L., Tselishcheva L.G. 2011. [To the spider fauna (Aranei) of "Nurgush" Reserve] // Trudy gosudarstvennogo prirodnogo zapovednica "Nurgush". Tom 1. Kirov, OOO "Tipografiya "Staraya Vyatka". P. 38-70 [in Russian].

Krasnobaev Yu.P. 2004. [Catalogue of spiders (Aranei) of the Middle Volga region]. Samara. 213 pp. [in Russian]

Mikhailov K.G., Trushina E.E. 2013. On the spider fauna (Arachnida: Aranei) of the Mordovian State Reserve, Russia: preliminary results // Arthropoda Selecta. Vol.22. No.2. P.189-196.

Sozontov A.N. 2012a. [First record of the wasp spider Argiope bruennichi (Aranei, Araneidae) from Udmurt Rebublic] // Vest- nik Udmurtskogo Universiteta. Seriya "Biologiya. Nauki o Zemle". Iss.4. P.151-152 [in Russian, with English summary]. Sozontov A.N. 2012b. [Some finds of spiders (Arachnida, Aranei) from Udmurt Republic interesting from the point of view to biogeography] // Problyemy prikladnoy i regionalnoy biogeografii (g. Izhevsk, 8-12 okt. 2012 g.): materyaly vserossiyskoy nauchno-prakticheskoy konferentsyi s mezhdunarodnym uchastiem. Izhevsk: Izd-vo "Udmurtskiy Universitet". P.246-251 [in Russian].

Sozontov A.N. 2013. [Spiders (Arachnida, Aranei) of Udmurt Republic: history and prospect of studies] // Vestnik Udmurtskogo Universiteta. Seriya "Biologiya. Nauki o Zemle". Iss.3. P.5157 [in Russian, with English summary].

Sozontov A.N., Dedyukhin S.V. 2012. [The Black Eresus - Eresus kollari Rossi, 1846 (= E. cinnaberinus (Olivier, 1787); E. niger (Petagna, 1787))] // Krasnaya Kniga Udmurtskoy Respubliki. Izd. 2-e. Cheboksary: Perfectum. P.22.[in Russian].

Sozontov A.N., Esyunin S.L. 2012. On the spider fauna (Arachnida: Aranei) of Udmurt Republic // Arthropoda Selecta. Vol. 21. No. 1. P. 85-95.

Zhu M.S., Song D.X., Zhang J.X., 2003. Fauna Sinica. Invertebrata. Vol.35. Arachnida: Araneae: Tetragnathidae. Beijing: Science Press. 418 pp.

Zubko T.L. 2001. [Spider - Aranei] // Krasnaya Kniga Udmurtskoy Respubliki. Izhevsk. P.7-12 [in Russian].

Zubko T.L., Roshchinenko V.I. 1981. [To the spider fauna of some regions of Udmurtian ASSR] // [Fauna i ekologiya zhivontykh Udmurtskoy ASSR i prilezhashchikh raionov. Izhevsk: Udmurtskiy Univ. Press. P.48-57 [in Russian].

Responsible editor D.V. Logunov 